\title{
MiR-212-5p Suppresses the Epithelial- Mesenchymal Transition in Triple-Negative Breast Cancer by Targeting Prrx2
}

\author{
Zhi-Dong Lva, bong-Xia Yang ${ }^{a} \quad$ Xiang-Ping Liuc Li-Ying Jin ${ }^{d} \quad$ Xin-Gang Wang ${ }^{a}$ \\ Zhao-Chuan Yange Dong Liu ${ }^{\mathrm{a}}$ Jiao-Jiao Zhao ${ }^{\mathrm{a}}$ Bin Kong ${ }^{\mathrm{a}}$ Fu-Nian Lia \\ Hai-Bo Wang ${ }^{\text {a }}$ \\ ${ }^{a}$ Center of Diagnosis and Treatment of Breast Disease, The Affiliated Hospital of Qingdao \\ University, Qingdao; 'Department of Breast Surgery, Qilu Hospital of Shandong University, Jinan; \\ 'Central Laboratory of Molecular Biology, The Affiliated Hospital of Qingdao University, Qingdao; \\ ${ }^{d}$ Cerebrovascular Disease Research Institute, The Affiliated Hospital of Qingdao University, Qingdao; \\ eDepartment of Child Health Care, The Affiliated Hospital of Qingdao University, Qingdao, P.R. China
}

\section{Key Words}

Mir-212-5p • Triple-negative breast cancer • Prrx2 • Epithelial-to -mesenchymal transition

\begin{abstract}
Background/Aims: Triple-negative breast cancer (TNBC) is the most aggressive breast cancer subtype. Our study investigated the functional role of miR-212-5p in TNBC. Methods: Realtime PCR was used to quantify miR-212-5p expression levels in 30 paired TNBC samples and adjacent normal tissues. Wound healing and Transwell assays were used to evaluate the effects of miR-212-5p expression on the invasiveness of TNBC cells. Luciferase reporter and Western blot assays were used to verify whether the mRNA encoding Prrx 2 is a major target of miR-212-5p. Results: MiR-212-5p was downregulated in TNBC, and its expression levels were related to tumor size, lymph node status and vascular invasion in breast cancer. We also observed that the miR-212-5p expression level was significantly correlated with a better prognosis in TNBC. Ectopic expression of miR-212-5p induced upregulation of E-cadherin expression and downregulation of vimentin expression. The expression of miR212-5p also suppressed the migration and invasion capacity of mesenchymal-like cancer cells accompanied by a morphological shift towards the epithelial phenotype. Moreover, our study observed that miR-212-5p overexpression significantly suppressed Prrx2 by targeting its 3'-untranslated region (3'-UTR) region, and Prrx2 overexpression partially abrogated miR-212-5p-mediated suppression. Conclusions: Our study demonstrated that miR-212-5p inhibits TNBC from acquiring the EMT phenotype by downregulating Prrx2, thereby inhibiting cell migration and invasion during cancer progression.




\section{Introduction}

Triple-negative breast cancer (TNBC) is a highly aggressive tumor subtype associated with poor prognosis, in which mortality and morbidity arise from distant metastases, rather than from the primary tumor [1]. The epithelial-to-mesenchymal transition (EMT) is crucial in tumor progression, as it allows tumor cells to escape from the primary tumor to invade adjacent tissues and migrate to distant regions [2]. EMT is a key step in local invasion and distant metastasis and is important for enabling breast cancer to resist targeted treatment [3].

Aberrantly expressed microRNA (miRNA), including those regulating EMT and cancer metastasis, are common in many cancers and are important for tumorigenesis to develop and progress $[4,5]$. MiR-212, located on chromosome 17p13.3, is deregulated in several human cancers [6, 7]. MiR-212 suppresses tumors in non-small cell lung cancer and gastric carcinoma [8]. However, other studies suggest that miR-212 exhibits oncogenic properties in colorectal, prostate and pancreatic cancers $[9,10]$. Therefore, the biological functions of miR-212 are cancer-type specific, partly resulting from the different cellular contexts of various tumors. Importantly, the miR-212-5p expression level and its role in breast cancer remain undetermined.

Prrx2 is a newly identified EMT-inducer conferring migratory and invasive properties [11]. Prrx2 is encoded by the Prrx2 gene, which belongs to the paired class of homeobox genes [12]. Prrx2 and Prrx1, paired-related homeobox transcription factors, are highly expressed in undifferentiated mesenchymal cells during embryogenesis and are critical for normal morphogenesis in the craniofacial region, limbs and vascular system [13]. A recent study reported that Prrx2 works as a TGF- $\beta$-induced factor that enhances breast cancer invasion and migration [14]. Our previous study revealed that Prrx2 expression was associated with tumor size, lymph node metastasis, TNM stages and poor prognosis in breast cancer. Furthermore, silencing Prrx2 may suppress the proliferation, invasion and migration of breast cancer by reversing the EMT phenotypes by downregulating the Wnt/ $\beta$-catenin signaling pathway [15]. Therefore, Prrx2 is an important contributor in breast cancer development. However, the regulatory mechanisms of Prrx2 expression in TNBC are poorly understood.

In this study, we provided experimental evidence that miR-212-5p is essential for the EMT and invasive phenotype of TNBC. Moreover, we revealed that a novel post-transcriptional regulatory mechanism of Prrx2 expression is mediated by miR-212-5p.

\section{Materials and Methods}

\section{Clinical samples and data}

Thirty pairs of TNBC and corresponding paired normal adjacent tissues were obtained from patients who underwent modified radical mastectomy at the Affiliated Hospital of Qingdao University. Tissues were diagnosed by histopathology. The matched non-cancerous adjacent tissues were harvested at least $5 \mathrm{~cm}$ away from the tumor site. No patients received radiotherapy or chemotherapy before surgery. Enrolled patients were divided into different subgroups based on miR-212-5p expression. Clinical features and survival information were compared between subgroups to determine the clinical significance and prognostic value of miR-212-5p.

\section{Cell cultures and transfection}

Three TNBC lines, MDA-MB 231 (PTEN wild-type, p53 mutant, and BRCA1 wild-type), MDA-MB-468 (PTEN null, p53 mutant, and BRCA1 wild-type), and HCC-1937 (PTEN null, p53 mutant, and BRCA1 mutant) and a normal mammary epithelial cell line (MCF-10A) were obtained from the Cancer Research Institute of Beijing, China. Cells were maintained per the supplier's instructions. Before the experiment, all cell lines were authenticated with short tandem repeat DNA profiling and were free of mycoplasma infection. 


\section{Cellular Physiology Cell Physiol Biochem 2017;44:1785-1795 \\ \begin{tabular}{l|l|l} 
and Biochemistry & $\begin{array}{l}\text { DOI: 10.1159/000485785 } \\
\text { Published onlIne: December 06, } 2017\end{array}$ & $\begin{array}{l}\text { C) } 2017 \text { The Author(s). Published by S. Karger AG, Basel } \\
\text { www.karger.com/cpb }\end{array}$
\end{tabular}}

Lv et al.: MiR-212-5p Inhibits EMT in Triple-Negative Breast Cancer

The miR-212-5p mimics were transfected at working concentrations using Lipofectamine 2000 reagent (Invitrogen, Carlsbad, CA, USA). Full-length Prrx2 cDNA was purchased from GeneCopoeia ${ }^{\mathrm{TM}}$ (GeneCopoeia, Inc., Rockville, MD, USA) and subcloned into the eukaryotic expression vector, pcDNA3.1 (+).

\section{Western blot analysis}

Denatured protein was separated on an SDS-polyacrylamide gel, transferred to a Hybond membrane, and blocked overnight in 5\% skim milk in TBST. For immunoblotting, the membrane was incubated overnight at $4^{\circ} \mathrm{C}$ with anti-Prrx2, anti-E-cadherin, and anti-vimentin antibodies (Santa Cruz, CA, USA; 1:500), followed by horseradish peroxidase-conjugated secondary antibody (Boster, Wuhan, China). Protein bands were visualized by ECL chemiluminescence (ECL New England Biolabs, USA),

\section{MTT proliferation assay}

Approximately, $5 \times 10^{3}$ cells were seeded into 96-well culture plates, incubated with $20 \mu \mathrm{L}$ MTT (10 $\mathrm{mg} / \mathrm{ml}$ ) for $4 \mathrm{~h}$ at $37^{\circ} \mathrm{C}$, and $200 \mu \mathrm{L}$ of dimethyl sulfoxide (DMSO) was pipetted to solubilize the formazan product for $20 \mathrm{~min}$ at room temperature. Optical density was determined using a spectrophotometer at a wavelength of $570 \mathrm{~nm}$.

\section{Luciferase reporter assay}

The luciferase reporter assay was performed as described previously [15]. Cell extracts were prepared $48 \mathrm{~h}$ after transfection, and luciferase activity was determined by the Dual-Luciferase Reporter Assay system (Promega, Madison, WI, USA).

\section{Quantitative real-time PCR}

Briefly, 10 ng of total RNA was reversed transcribed into cDNA using TaqMan miRNA hsa-miR-125bspecific primers (Applied Biosystems). Real-time PCR was performed using a TaqMan MicroRNA Reverse Transcription Kit (Applied Biosystems). RNA U6 was used as an endogenous control in each sample.

\section{Wound healing assay}

A wound healing assay was performed to examine cell migration. Briefly, after the cells grew to $90 \%$ confluence in six-well plates, a single scratch wound was generated with a $200-\mu \mathrm{L}$ disposable pipette tip. Scratch wounds were photographed over a $24 \mathrm{~h}$ period using a Nikon inverted microscope with an attached digital camera, and the wound widths were measured using Image software (Version 2.2.1, Nicon-BioImage Ltd., Japan).

\section{Migration and invasion assays}

Migration and invasion assays were performed in Boyden chambers as described previously [15]. Cancer cells were stained and counted microscopically. All experiments were performed at least twice in triplicate.

\section{Mouse xenograft model}

A total of $2 \times 10^{6}$ MDA-MB-231 cells infected with miR-212-5p or scramble viruses were propagated and inoculated subcutaneously into the dorsal flanks of female BALB/c nude mice (SLAC, Shanghai, China). Tumor size was measured every 7 days. After 42 days, the mice were sacrificed, necropsies were performed, and the tumors were weighed. The tumor volumes were determined by the following formula: $A \times \mathrm{B}^{2} / 2$, where $\mathrm{A}$ is the largest diameter and $\mathrm{B}$ is the diameter perpendicular to $\mathrm{A}$.

\section{Statistical analysis}

Comparisons between groups were analyzed using $t$-tests and $\chi^{2}$ tests. Overall survival curves and disease-free curves were plotted per the Kaplan-Meier method, and the log-rank test was used for comparison. Survival was counted from the date of surgery. Variables with $P<0.05$ by univariate analysis were used in a subsequent multivariate analysis based on the Cox proportional hazards model. Statistical analyses were performed using SPSS 18.0 software. 


\section{Results}

miR-212-5p expression in TNBC tissues and its association with clinicopathologic features

To determine the expression status of miR-212-5p in TNBC, we initially compared miR-212-5p expression in 30 pairs of TNBC and adjacent non-tumor tissues. miR-212-5p expression in the TNBC tissues was significantly lower than in the matched tumor-adjacent tissues $(P=0.003$, Fig. $1 \mathrm{~A})$. Furthermore, we evaluated the relative expression of miR-212$5 \mathrm{p}$ in a normal breast epithelial cell line (MCF-10A) and three basal-like breast cancer cell lines (MDA-MB-468, HCC-1937 and MDA-MB-231). Reduced expression of miR-212-5p was observed in all three basal-like breast cancer cell lines compared to MCF-10A $(P<0.01$, Fig. 1B). Notably, miR-212-5p expression in HCC-1937 and MDA-MB-231 cells was lower than in MDA-MB-468 cells. We then determined the potential clinicopathological implications of altered miR-212-5p expression in 125 patients. The clinical samples were divided into low- and highexpression groups based on whether the miR-212$5 \mathrm{p}$ expression scores were greater or lower than the median. We analyzed the relationship between miR212-5p expression and patient clinicopathological parameters. The results showed that low miR-212$5 \mathrm{p}$ expression was closely related to TNBC pathological parameters, including tumor size, lymph node status and vascular invasion (Table 1).

Table 1. Correlation of miR-212-5p expression with patient's clinical and pathological characteristics $\left({ }^{*} \mathrm{P}<0.05\right)$

\begin{tabular}{|c|c|c|c|c|}
\hline \multirow{2}{*}{ Clinicopathological variables } & \multirow[t]{2}{*}{ Cases } & \multicolumn{2}{|c|}{ miR-212-5p expression level } & \multirow[b]{2}{*}{$P$ value } \\
\hline & & No. of low expression & No. of high expression & \\
\hline Age (years) & & & & \\
\hline$\leq 45$ & 45 & 26 & 19 & 0.871 \\
\hline$>45$ & 80 & 48 & 32 & \\
\hline Tumor size $(\mathrm{cm})$ & & & & \\
\hline$\leq 2$ & 46 & 21 & 25 & $0.019^{*}$ \\
\hline$>2$ & 79 & 53 & 26 & \\
\hline Differentiation grade & & & & \\
\hline Well-moderate & 57 & 34 & 23 & 0.925 \\
\hline Poor-undifferentiation & 68 & 40 & 28 & \\
\hline Vascular invasion & & & & \\
\hline Negative & 57 & 25 & 32 & $0.012^{*}$ \\
\hline Positive & 68 & 49 & 19 & \\
\hline Lymph node status & & & & \\
\hline Negative & 62 & 30 & 32 & $0.015^{*}$ \\
\hline Positive & 63 & 44 & 19 & \\
\hline Ki67 & & & & \\
\hline$\leq 20 \%$ & 75 & 45 & 30 & 0.824 \\
\hline$>20 \%$ & 50 & 29 & 21 & \\
\hline
\end{tabular}

Fig. 1. miR-212-5p expression levels are frequently downregulated in TNBC. (A) Expression levels of miR-212-5p in 30 paired TNBC specimens and their corresponding paired adjacent normal tissues. (B) Expression levels of miR212-5p determined by qRT-PCR in three basal-like breast cancer cell lines (HCC-1937, MDA-MB-468,

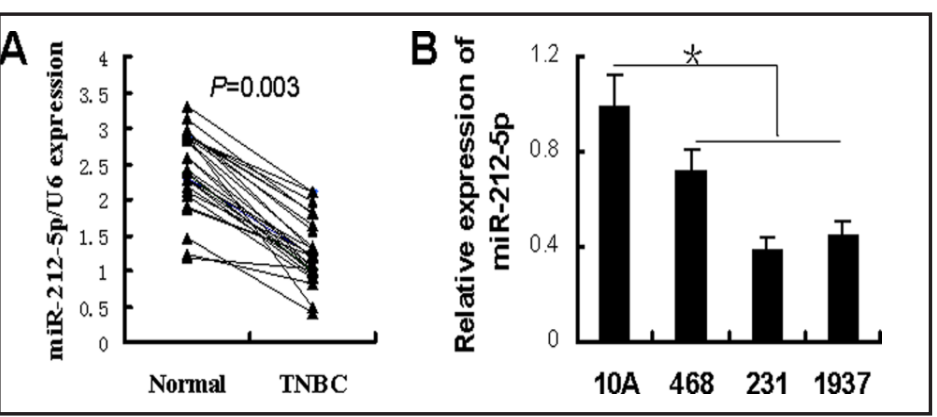
and MDA-MB-231) and a normal epithelial cell line (MCF-10A). miR-212-5p expression was normalized based on U6 RNA expression. Error bars represent the standard deviations from triplicates of one representative experiment. $* \mathrm{P}<0.05$. 
Decreased miR-212-5p levels are correlated with poor clinical outcomes

To further analyze the significance of miR-212-5p on clinical prognosis, a Kaplan-Meier survival analysis was performed using the patient disease-free survival and overall survival. The results demonstrated that the 10-year disease-free survival and overall survival of 125 patients were $70.8 \%$ and $75.7 \%$, respectively. When the patients were stratified by miR-212$5 \mathrm{p}$ expression status, the 10-year disease-free survival rates for high miR-212-5p expression and low miR-212-5p expression patients were $78.5 \%$ and $54.9 \%$, respectively $(P=0.001)$. Overall survival rates for high miR-212-5p expression and low miR-212-5p-expression patients were $81.9 \%$ and $62.3 \%$, respectively $(P=0.002)$ (Fig. 2). Univariate and multivariate survival analysis showed that higher histological stage, positive lymph node status, and low miR-212-5p expression were significantly associated with reduced disease-free survival and overall survival in the 125 patients (Table 2 ).

Table 2. Univariate and multivariate survival analysis of miR-212-5p expression in breast cancer $\left({ }^{*} \mathrm{P}<0.05\right)$

\begin{tabular}{lcccc}
\hline & \multicolumn{2}{c}{ Univariate analyses } & \multicolumn{2}{c}{ Multivariate analyses } \\
& HR $(95 \% \mathrm{CI})$ & P value & HR $(95 \% \mathrm{CI})$ & P value \\
\hline Age & $0.915(0.530-1.582)$ & 0.752 & $1.126(0.579-2.190)$ & 0.727 \\
Menopausal & $0.815(0.471-1.409)$ & 0.464 & $1.380(0.614-3.099)$ & 0.435 \\
Tumor size & $1.373(0.795-2.371)$ & 0.256 & $1.630(0.868-3.062)$ & 0.129 \\
Lymph node status & $2.330(2.356-4.005)$ & $0.002^{*}$ & $2.181(1.212-3.925)$ & $0.009^{*}$ \\
Histological grade & $1.715(1.013-2.906)$ & $0.045^{*}$ & $1.879(1.031-3.427)$ & $0.027^{*}$ \\
& & & & \\
miR-212-5p & $0.484(0.228-2.026)$ & $0.049^{*}$ & $0.344(0.132-0.884)$ & $0.027^{*}$ \\
\hline
\end{tabular}
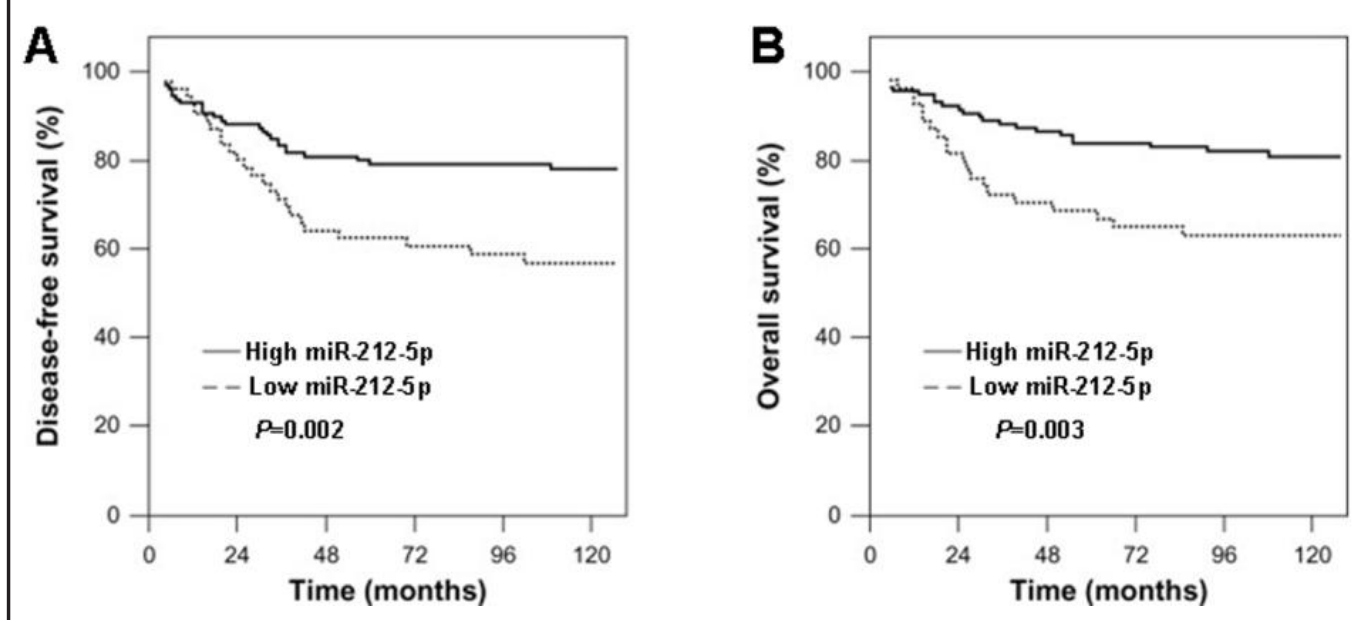

Fig. 2. Prognostic value of miR-212-5p for TNBC patients assessed by Kaplan-Meier analysis. Low miR212-5p expression is associated with poor prognosis in TNBC patients. Disease-free survival (A) and overall survival (B) per the miR-212-5p expression level in 125 patients. 


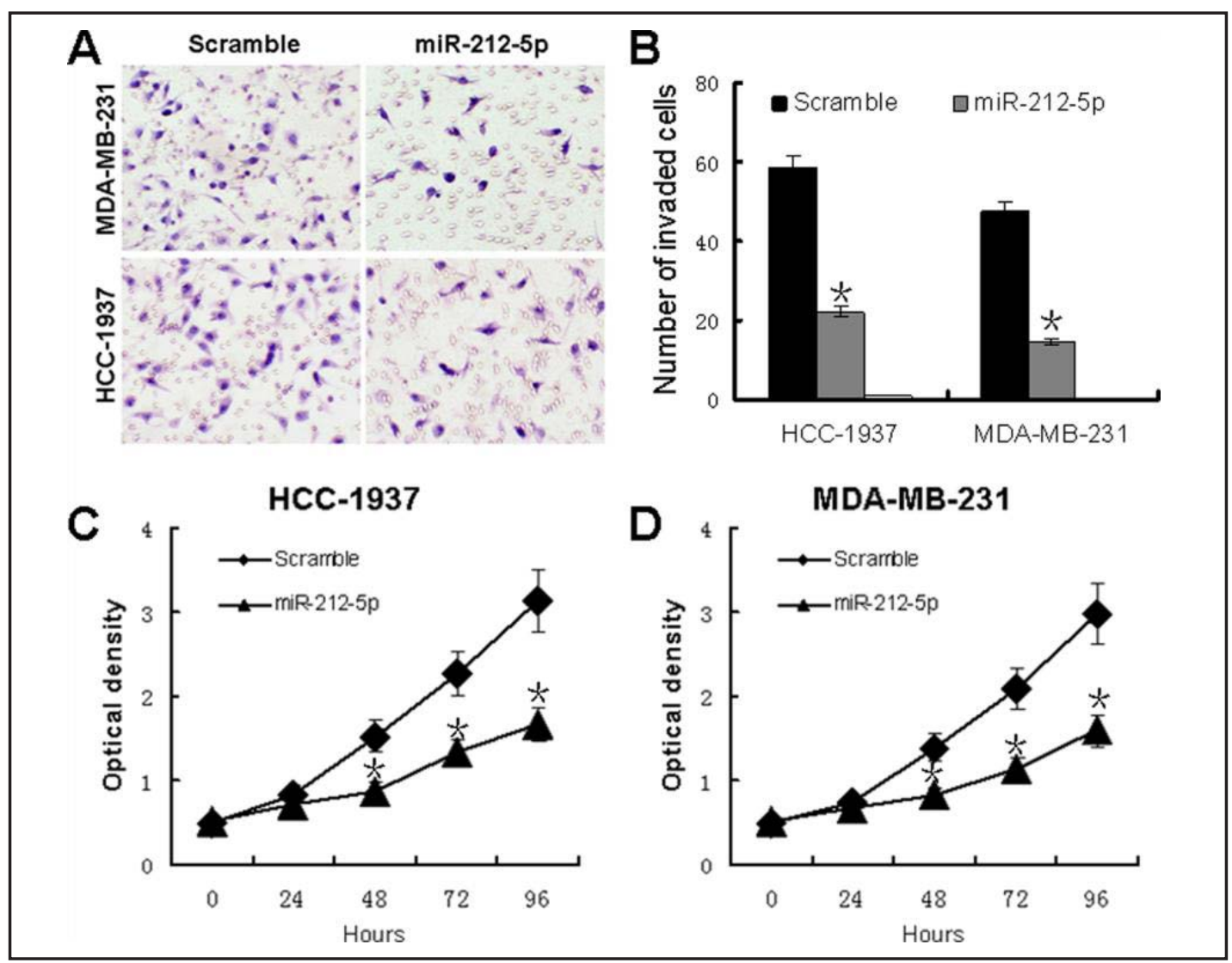

Fig. 3. miR-212-5p overexpression inhibits cell proliferation and invasion in vitro. MDA-MB-231 and HCC1937 cells infected with miR-212-5p or scramble lentivirus. (A) The invasion assay was measured through Transwell assays with Matrigel. (B) The results from three separate assays were averaged and graphed. The growth of HCC-1937 (C) and MDA-MB-231 (D) cells infected with miR-212-5p or the scramble lentivirus was assayed. The results from three separate assays were averaged and graphed. ${ }^{*} \mathrm{P}<0.05$.

Ectopic expression of miR-212-5p suppressed proliferation and invasion ability of breast cancer in vitro

To determine whether miR-212-5p functionally behaved as a tumor suppressor, we demonstrated that upregulated miR-212-5p expression inhibited the invasive capacity of breast cancer cells (Fig. 3A, B). We also demonstrated that overexpression of miR-212-5p significantly suppressed breast cancer cell proliferation (Fig. 3C, D).

miR-212-5p suppressed tumorigenesis and metastasis in vivo

To directly evaluate the role of miR-212-5p in tumor formation and growth in vivo, a xenograft model of human TNBC cells in nude mice was adopted. Briefly, MDA-MB-231 cells infected with miR-212-5p or the scramble lentivirus were injected subcutaneously into each flank of the nude mice. After the cells were injected, the tumor volume was monitored every 7 days, and tumor growth curves were plotted accordingly. All mice were then sacrificed to harvest the xenograft. The mean volume and weight of the tumors generated from the miR212-5p overexpression group were significantly lower than the control group $(P<0.05)$ (Fig. $4 A, B, C)$. We then investigated the effect of miR-212-5p on tumor metastasis in vivo. MDAMB-231 cells infected with miR-212-5p or the scramble lentivirus were transplanted into the nude mice via tail vein injection. After 60 days, the mice were anesthetized, and their lungs were dissected. As shown in Fig. 4D, significantly fewer macroscopic lung metastases were observed in the mice that received cells infected with miR-212-5p $(P<0.05)$. 
Fig. 4. miR-212$5 p$ inhibits TNBC growth and metastasis in vivo. Tumor growth in mouse xenograft models. (A) MDAMB-231 cells infected with miR212-5p lentivirus or scramble were injected subcutaneously into nude mice. (B) Tumor volume was measured every 7 days. (C) After 42 days, the mice were sacrificed, necropsies were performed, and the tumors were weighed. Tumor metasta-

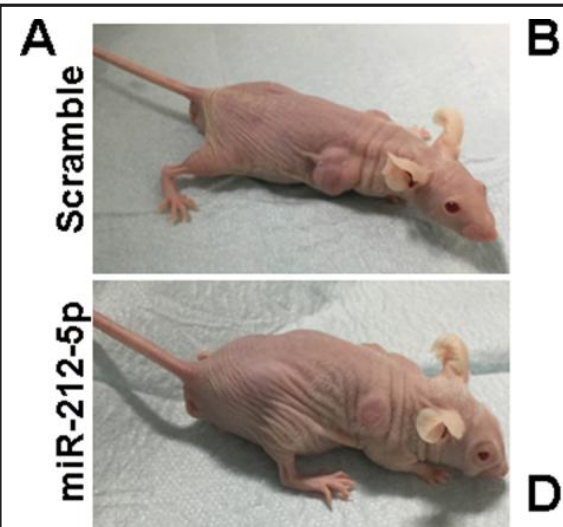

C

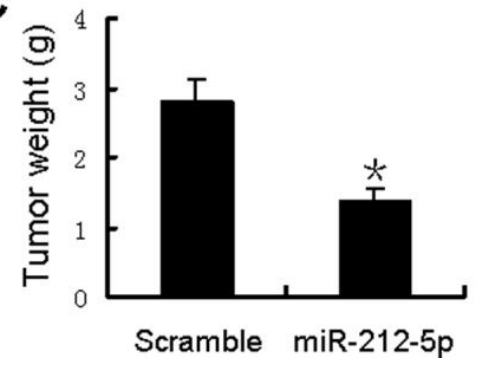

B
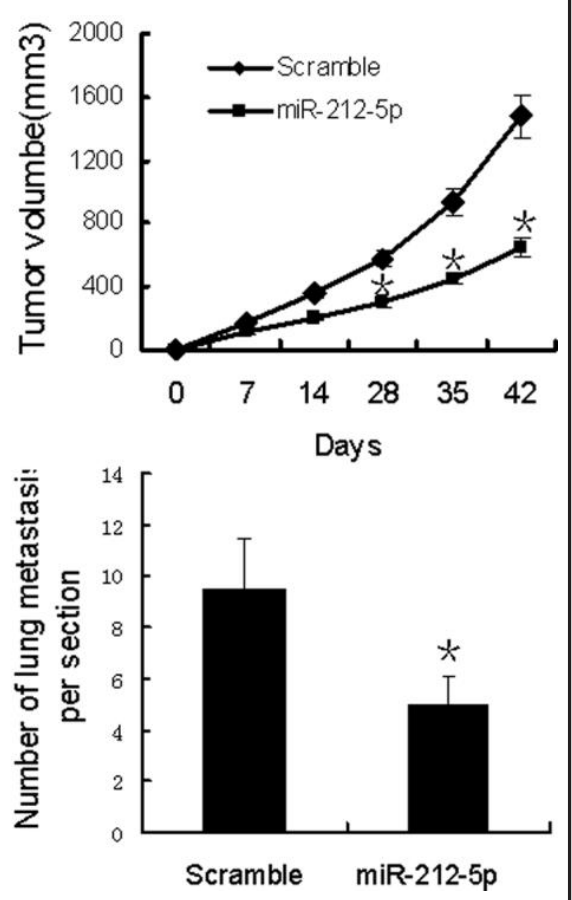

sis in mouse xenograft models. MDA-MB-231 cells overexpressing miR-212-5p or scramble were injected into the tail vein of nude mice. After 60 days, the mice were sacrificed. (D) The disseminated nodules were evaluated. Each group had eight mice. ${ }^{*} \mathrm{P}<0.05$.

\section{miR-212-5p inhibited the EMT process of TNBC}

To investigate the effects of miR-212-5p up-regulation on the EMT phenotype in breast cancer cells, we measured the relative protein expression of EMT markers by Western blot analysis. Upregulated miR-212-5p expression in MDA-MB-231 cells led to significantly increased E-cadherin expression and decreased vimentin expression in the cells (Fig. 5A, B). Additionally, cells with overexpressed miR-212-5p exhibited epithelial morphology (Fig. 5C).

miR-212-5p directly targeted the Prrx2 3. UTR

We performed a bioinformatics analysis using TargetScan and Pictar, which predicted that miR-212-5p may target the Prrx2 3, UTR region. Perfect base pairing was observed between the seed sequence of mature miR-212-5p and the 3. UTR of Prrx2 mRNA, and these seed sequences were conserved across species (Fig. 6A). To determine whether the 3. UTR of Prrx2 mRNA is a functional target of miR-212-5p in breast cancer cells, either the target sequence of Prrx2 3, UTR (wt 3, UTR) or the mutant sequence (mt 3, UTR) were cloned into a luciferase reporter vector (Fig. 6B). Thereafter, HEK293 cells were transfected with a wt or mt 3. UTR vector and miR-212-5p mimics. Luciferase activity decreased significantly compared with the control. As shown in Fig. 6C, ectopic overexpression of miR-212-5p inhibited Prrx2 expression $(P=0.002)$, while a miR-212-5p inhibitor restored Prrx2 expression $(P=0.010)$.

Exogenous Prrx2 reversed the inhibitory effects of miR-212-5p on breast cancer cells in vitro

We examined whether miR-212-5p regulates cell behaviors by downregulating Prrx2. We transfected pcDNA3.1-Prrx2 into the miR-212-5p-overexpressed breast cancer cell line, MDA-MB-231. Western blot analysis showed that Prrx2 reversed the expression of EMT-related genes caused by overexpressed miR-212-5p (Fig. 7A). We then tested whether 


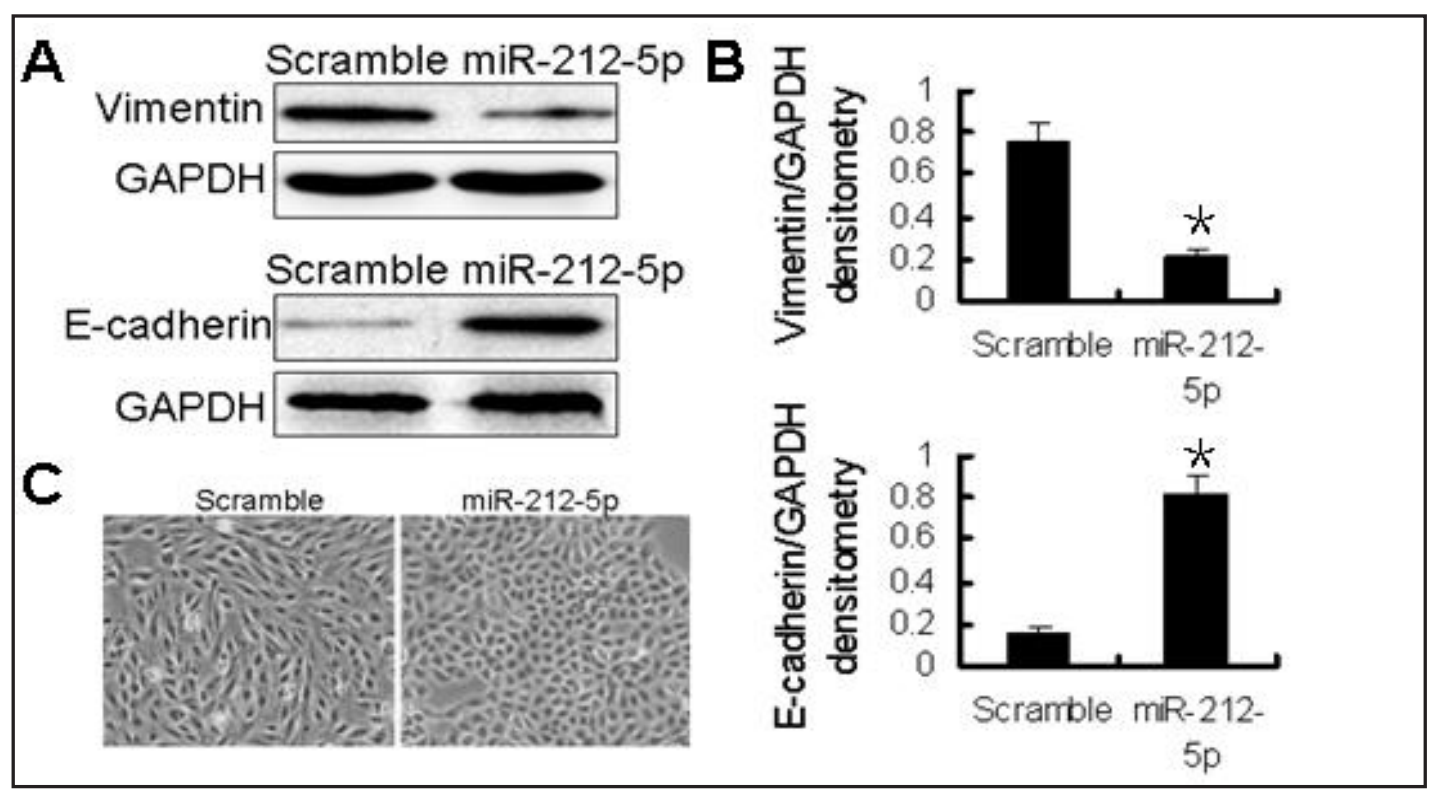

Fig. 5. Upregulation of miR-212-5p altered the EMT phenotype in breast cancer cells. Breast cancer cells (MDA-MB-231) were transfected with miR-212-5p mimics or negative control mimics for $48 \mathrm{~h}$. (A) Western blot analysis of E-cadherin and vimentin expression in MDA-MB-231 cells. (B) Data were expressed as the mean \pm SD of three independent experiments relative to GAPDH. (C) Morphological changes in the breast cancer cells were observed by phase-contrast microscopy $(100 \times) .{ }^{*} \mathrm{P}<0.05$.

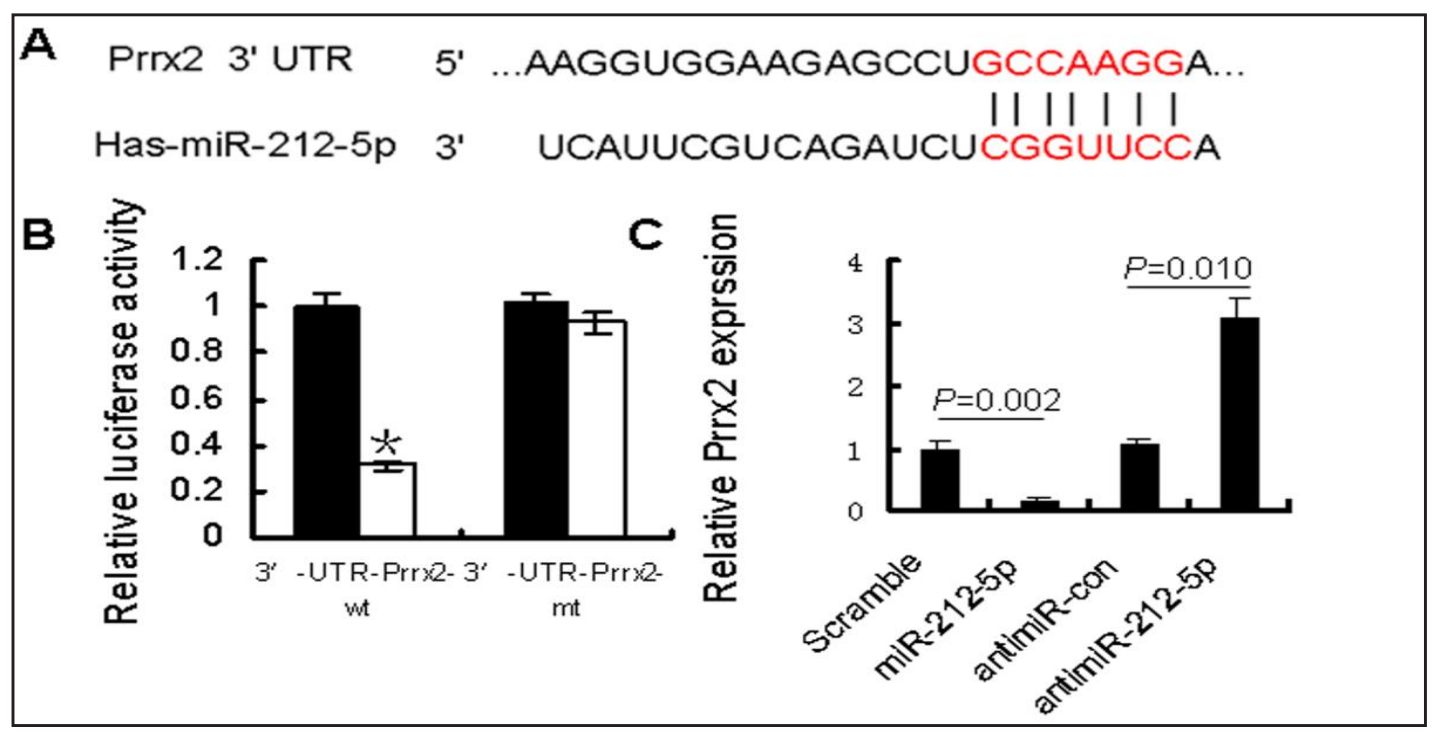

Fig. 6. Oncogene Prrx2 was specifically targeted by miR-212-5p. (A) The predicted binding sequences for miR-212-5p within the Prrx2 3 UTR. Seed sequences are highlighted. (B) Luciferase activity assays using a luciferase reporter with wild-type or mutant human Prrx2 3 UTR were performed after co-transfection of miR-212-5p mimics or control into HEK293 cells. mt 3 UTR increased significantly compared with wt 3' UTR. (C) Prrx2 expression was determined in MDA-MB-231 that stably overexpressed miR-212-5p and cells transfected with miR-212-5p inhibitors or anti-miR-control by Western blot analysis. ${ }^{*} \mathrm{P}<0.05$.

restoring Prrx2 could reverse the miR-212-5p-mediated inhibition of breast cancer cell invasion. As shown in Figs. 7B, C, and D, Prrx2 over-expression using cDNA without a 3. UTR partially abrogated the miR-212-5p-mediated suppression of breast cancer cell migration and invasion $(P<0.05)$.

\section{KARGER}




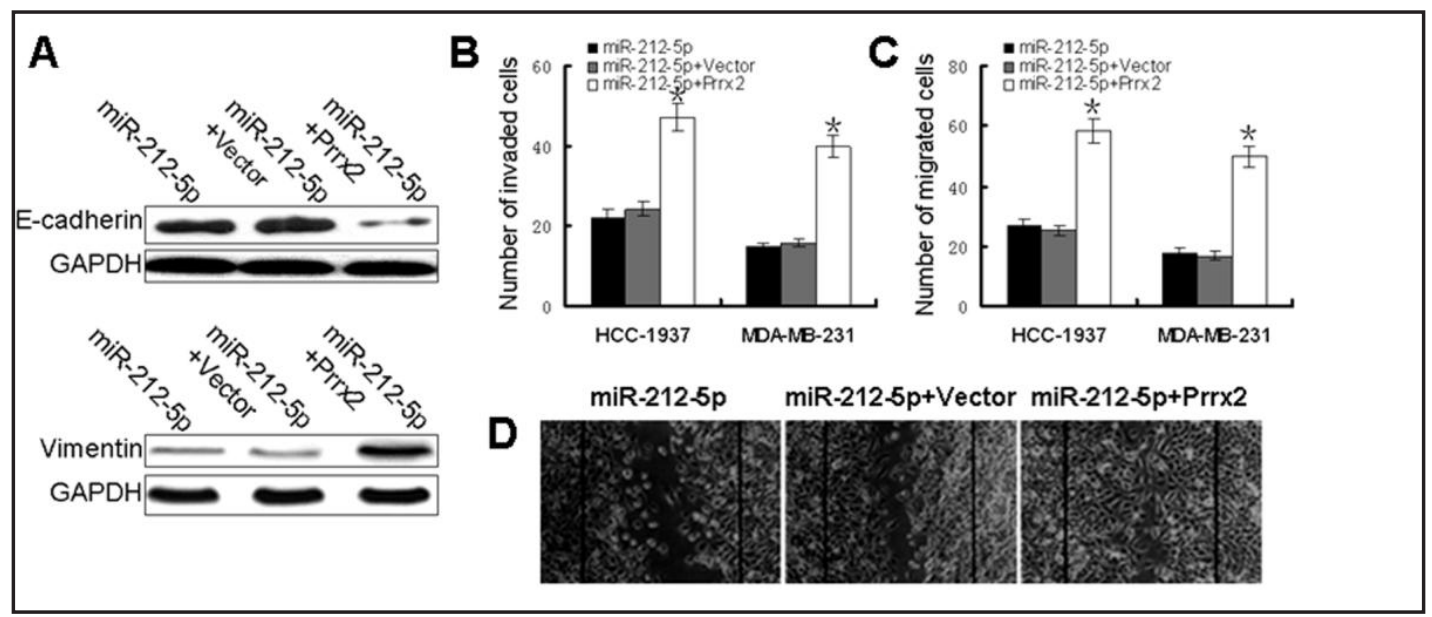

Fig. 7. Overexpressing Prrx2 reverses the inhibitory effects of miR-212-5p on breast cancer cells. (A) Western blot was used to analyze the Prrx2 and EMT-related genes, E-cadherin and vimentin, in miR-212-5pvector co-transfected cells or miR-212-5p Prrx2 co-transfected cells compared with the control group. Transwell assay revealed that the reduced migration (B) and invasion (C) caused by overexpressing miR-212-5p could be reversed by introducing Prrx2. (D) A wound healing assay indicated that the reduced migration caused by overexpressing miR-212-5p could be reversed by introducing Prrx2 (40×). ${ }^{*} \mathrm{P}<0.05$.

\section{Discussion}

miRNA regulates up to $30 \%$ of all human genes and controls many cellular processes $[16,17]$. Recent studies have shown that miRNA is deregulated in various cancers, and miRNA expression is relevant to the diagnosis and prognosis of many diverse tumors [18, 19]. Various publications have associated miRNA with cancer; however, the relationship between miR-212-5p and TNBC remains unknown. In this study, we demonstrated that miR$212-5 p$ is significantly downregulated in TNBC tissues. We also found that altered miR-212$5 p$ expression levels are associated with tumor size, lymph node status and vascular invasion in breast cancer. We demonstrated a significant correlation between higher miR-212-5p expression and better prognosis in TNBC. Using wound healing and Transwell assays, we revealed that upregulating miR-212-5p in HCC-1937 and MDA-MB-231 cells suppressed the migration and invasion of these TNBC strains. Moreover, Kaplan-Meier survival analysis revealed that breast cancer patients whose primary tumors displayed low miR-212-5p expression had a shorter disease-free and overall survival. The observed aberrant expression of miR-212-5p suggested that miR-212-5p suppresses TNBC tumors.

New evidence suggests that the EMT is important for tumor metastasis and recurrence $[20,21]$. Understanding the molecular mechanisms that regulate the EMT process is crucial for improving breast carcinoma treatment [22, 23]. Our study showed that ectopic expression of miR-212-5p in TNBC cells impaired their invasion, proliferation and growth. High expression of miR-212-5p resulted in increased E-cadherin expression and decreased vimentin expression. Moreover, in vivo data demonstrated that miR-212-5p inhibited tumor growth and metastasis. These in vitro and in vivo data further indicate that miR-212-5p inhibits cell migration, invasion and the EMT phenotype in TNBC. However, miR-212-5p mechanisms in the EMT and their therapeutic potential remain unclear. Various studies have shown that fatty acid synthase (FAS) and stearoyl-CoA desaturase-1 (SCD1) are potential downstream target genes of miR-212-5p [24, 25]. Interestingly, we characterized the role of miR-212-5p in regulating Prrx2 in TNBC. We performed a bioinformatics analysis using TargetScan and Pictar and predicted that miR-212-5p may target the Prrx2 3. UTR region. Prrx2 is a transcription coactivator, enhancing the DNA-binding activity of serum response factors. A recent study reported that Prrx2 works as a TGF- $\beta$-induced factor that enhances invasion and migration in breast cancer. More importantly, our previous study showed that 
Lv et al.: MiR-212-5p Inhibits EMT in Triple-Negative Breast Cancer

silencing Prrx2 may suppress the proliferation, invasion and migration of breast cancer cells by reversing the EMT phenotypes by repressing the Wnt/ $\beta$-catenin signaling pathway $[15$, 26]. Here, Prrx 2 was identified as an important downstream target of miR-212-5p. Using a luciferase reporter assay, MiR-212-5p bound directly to the 3. UTR of Prrx2, containing a miR-212-5p-binding site. Upregulating miR-212-5p significantly reduced the Prrx2 protein level in MDA-MB-231. More importantly, we showed that overexpressing Prrx2 partially abrogated miR-212-5p-mediated suppression. These data suggest that miR-212-5p may inhibit MDA-MB-231 proliferation and metastasis by regulating Prrx2.

\section{Conclusion}

We revealed that miR-212-5p was downregulated in TNBC, and its expression level was associated with tumor size, lymph node status and vascular invasion in breast cancer. Furthermore, miR-212-5p inhibited EMT and metastasis in vitro and in vivo by downregulating Prrx2 in TNBC. These findings suggest that miR-212-5p suppresses tumors in TNBC development, and miR-212-5p can serve as a prognostic marker for breast cancer.

\section{Acknowledgements}

This study was supported by Natural Science Foundation of Shandong Province (No. ZR2017PH032), Higher Educational Science and Technology Program of Shandong Province (No.J17B092) and National Natural Science Foundation of China (No. 81302290 and 81700029).

\section{Disclosure Statement}

The authors declare that they have no competing interests exist.

\section{References}

1 Gao S, Wang Y, Wang M, Li Z, Zhao Z, Wang RX, Wu R, Yuan Z, Cui R, Jiao K, Wang L, Ouyang L, Liu R: MicroRNA-155, induced by FOXP3 through transcriptional repression of BRCA1, is associated with tumor initiation in human breast cancer. Oncotarget 2017;8:41451-41464.

-2 Yan K, Tian J, Shi W, Xia H, Zhu Y: LncRNA SNHG6 is Associated with Poor Prognosis of Gastric Cancer and Promotes Cell Proliferation and EMT through Epigenetically Silencing p27 and Sponging miR-101-3p. Cell Physiol Biochem 2017;42:999-1012.

3 Lee AF, Chen MC, Chen CJ, Yang CJ, Huang MS, Liu YP: Reverse epithelial-mesenchymal transition contributes to the regain of drug sensitivity in tyrosine kinase inhibitor-resistant non-small cell lung cancer cells. PLoS One 2017;12:e0180383.

-4 Hiramoto H, Muramatsu T, Ichikawa D, Tanimoto K, Yasukawa S, Otsuji E, Inazawa J: miR-509-5p and miR1243 increase the sensitivity to gemcitabine by inhibiting epithelial-mesenchymal transition in pancreatic cancer. Sci Rep 2017;7:4002.

5 Chang TH, Tsai MF, Gow CH, Wu SG, Liu YN, Chang YL, Yu SL, Tsai HC, Lin SW, Chen YW, Kuo PY, Yang PC, Shih JY: Upregulation of microRNA-137 expression by Slug promotes tumor invasion and metastasis of nonsmall cell lung cancer cells through suppression of TFAP2C. Cancer Lett 2017;402:190-202.

6 Jeong HS, Kim JY, Lee SH, Hwang J, Shin JW, Song KS, Lee S, Kim J: Synergy of circulating miR-212 with markers for cardiovascular risks to enhance estimation of atherosclerosis presence. PLoS One 2017;12:e0177809.

7 Jiang C, Wang H, Zhou L, Jiang T, Xu Y, Xia L: MicroRNA-212 inhibits the metastasis of nasopharyngeal carcinoma by targeting SOX4. Oncol Rep 2017;38:82-88. 


\section{Cellular Physiology Cell Physiol Biochem 2017;44:1785-1795 \begin{tabular}{l|l|l} 
and Biochemistry & DOI: 10.1159/000485785 & $\begin{array}{l}\text { (c) } 2017 \text { The Author(s). Published by S. Karger AG, Basel } \\
\text { www.karger.com/cpb }\end{array}$
\end{tabular}

8 Lu L, Zhang X, Zhang B, Wu J, Zhang X: Synaptic acetylcholinesterase targeted by microRNA-212 functions as a tumor suppressor in non-small cell lung cancer. Int J Biochem Cell Biol 2013;45:2530-2540.

-9 Yin Y, Song M, Gu B, Qi X, Hu Y, Feng Y, Liu H, Zhou L, Bian Z, Zhang J, Zuo X, Huang Z: Systematic analysis of key miRNAs and related signaling pathways in colorectal tumorigenesis. Gene 2016;578:177-184.

10 Wu Z, Zhou L, Ding G, Cao L: Overexpressions of miR-212 are associated with poor prognosis of patients with pancreatic ductal adenocarcinoma. Cancer Biomark 2017;18:35-39.

11 Higuchi M, Kato T, Yoshida S, Ueharu H, Nishimura N, Kato Y: Prrx1- and Prrx2-positive mesenchymal stem/ progenitor cells are involved in vasculogenesis during rat embryonic pituitary development. Cell Tissue Res 2015;361:557-565.

12 Ueharu H, Higuchi M, Nishimura N, Yoshida S, Shibuya S, Sensui K, Kato T, Kato Y: Expression of Krüppellike factor 6, KLF6, in rat pituitary stem/progenitor cells and its regulation of the Prrx2 gene. J Reprod Dev 2014;60:304-311.

13 Higuchi M, Yoshida S, Ueharu H, Chen M, Kato T, Kato Y: Prrx1 and Prrx2 distinctively participate in pituitary organogenesis and a cell-supply system. Cell Tissue Res 2014;357:323-335.

14 Juang YL, Jeng YM, Chen CL, Lien HC: Prrx2 as a novel TGF- $\beta$-induced factor enhances invasion and migration in mammary epithelial cell and correlates with poor prognosis in breast cancer. Mol Carcinog 2016;55:2247-2259.

15 Zhi-Dong Lv, Hai-Bo Wang, Xiang-Ping Liu, Li-Ying Jin, Ruo-Wu Shen, Xin-Gang Wang, Bin Kong, Hui-Li Qu, Fu-Nian Li, Qi-Feng Yang: Silencing of prrx2 inhibits the invasion and metastasis of breast cancer both in vitro and in vivo by reversing epithelial-mesenchymal transition. Cell Physiol Biochem 2017;42:1847 -1856.

16 Yu Z, Jian Z, Shen SH, Purisima E, Wang E: Global analysis of microRNA target gene expression reveals that miRNA targets are lower expressed in mature mouse and Drosophila tissues than in the embryos. Nucleic Acids Res 2007;35:152-164.

17 Xie T, Huang M, Wang Y, Wang L, Chen C, Chu X: MicroRNAs as Regulators, Biomarkers and Therapeutic Targets in the Drug Resistance of Colorectal Cancer. Cell Physiol Biochem 2016;40:62-76.

-18 Li S, Zhang H, Ning T, Wang X, Liu R, Yang H, Han Y, Deng T, Zhou L, Zhang L, Bai M, Wang X, Ge S, Ying G, Ba Y: MiR-520b/e Regulates Proliferation and Migration by Simultaneously Targeting EGFR in Gastric Cancer. Cell Physiol Biochem 2016;40:1303-1315.

19 Kanwal R, Plaga AR, Liu X, Shukla GC, Gupta S: MicroRNAs in prostate cancer: Functional role as biomarkers. Cancer Lett 2017;407:9-20.

20 Dong L, Ni J, Hu W, Yu C, Li H: Upregulation of Long Non-Coding RNA PlncRNA-1 Promotes Metastasis and Induces Epithelial-Mesenchymal Transition in Hepatocellular Carcinoma. Cell Physiol Biochem 2016;38:836-846.

-21 Liao TT, Yang MH: Revisiting epithelial-mesenchymal transition in cancer metastasis: the connection between epithelial plasticity and stemness. Mol Oncol 2017;11:792-804.

22 Neelakantan D, Zhou H, Oliphant MUJ, Zhang X, Simon LM, Henke DM, Shaw CA, Wu MF, Hilsenbeck SG, White LD, Lewis MT, Ford HL: EMT cells increase breast cancer metastasis via paracrine GLI activation in neighbouring tumour cells. Nat Commun 2017;8:15773.

-23 Zhu A, Li Y, Song W, Xu Y, Yang F, Zhang W, Yin Y, Guan X: Antiproliferative Effect of Androgen Receptor Inhibition in Mesenchymal Stem-Like Triple-Negative Breast Cancer. Cell Physiol Biochem 2016;38:10031014.

-24 YJ Guo, JJ Yu, CX Wang, K Li, B Liu, Y Du, F Xiao, SH Chen, FF Guo: miR-212-5p suppresses lipid accumulation by targeting FAS and SCD1. J Mol Endocrinol 2017;59:205-217.

25 Chen X, Wu J, Huang H, Ding Q, Liu X, Chen L, Zha X, Liang M, He J, Zhu Q, Wang S, Xia T: Comparative Profiling of Triple-Negative Breast Carcinomas Tissue Glycoproteome by Sequential Purification of Glycoproteins and Stable Isotope Labeling. Cell Physiol Biochem 2016;38:110-121.

-26 Yang N, Zhou TC, Lei XX, Wang C, Yan M, Wang ZF, Liu W, Wang J, Ming KH, Wang BC, Xu BL, Liu Q: Inhibition of Sonic Hedgehog Signaling Pathway by Thiazole Antibiotic Thiostrepton Attenuates the CD44+/CD24Stem-Like Population and Sphere-Forming Capacity in Triple-Negative Breast Cancer. Cell Physiol Biochem 2016;38:1157-1170. 